\title{
Dietary intake of vitamin D amongst UK adolescents
}

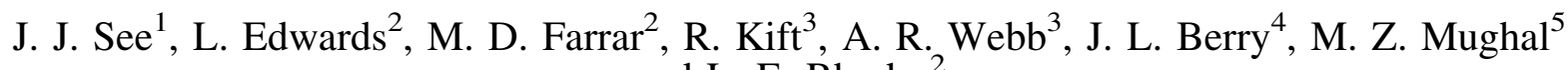 \\ and L. E. Rhodes ${ }^{2}$ \\ ${ }^{1}$ Manchester Medical School, University of Manchester, Manchester M13 9PL, UK, ${ }^{2}$ Photobiology Unit, Dermatology \\ Centre, University of Manchester, Salford Royal NHS Foundation Hospital, Salford M6 8HD, UK, ${ }^{3}$ School of Earth, \\ Atmospheric and Environmental Sciences, University of Manchester, Manchester M13 9PL, UK, ${ }^{4}$ Vitamin D Research \\ Laboratory, Institute of Human Development, University of Manchester, Manchester Royal Infirmary, Manchester M13 \\ 9WL, UK and ${ }^{5}$ Department of Paediatric Endocrinology, Royal Manchester Children's Hospital, Manchester M13 9WL, UK
}

Vitamin D is important during the adolescent bone growth spurt, when $\sim 50 \%$ of bone mineral accrual occurs, influencing present and future bone health ${ }^{(1)}$. Commonly known as the 'sunshine vitamin', vitamin D is predominately obtained through cutaneous synthesis after exposure to ultraviolet B (UVB) radiation in sunlight, whilst a smaller percentage is obtained via the diet ${ }^{(2)}$. However, at northerly latitudes, UVB is scarce during the winter months, and there is little information focusing on the oral vitamin D intake of adolescents. The main objectives of this study were to estimate dietary vitamin D intake in UK white Caucasian adolescents, and to determine whether the values meet the World Health Organisation (WHO) recommendation of $5 \mu \mathrm{g} / \mathrm{day}^{(3)}$ or the more recent Institute of Medicine (IOM) guidance of $15 \mu \mathrm{g} / \mathrm{day}^{(4)}$. A further aim was to compare adolescent intake with that of previously collected data from an adult white Caucasian sample.

This was a 1 year observational study of 124 healthy white Caucasian adolescents aged 12-15 years recruited from six schools in Greater Manchester. Adolescents completed a daily dietary record of seven vitamin D-containing food categories and intake of supplements for one week in each season and the average daily vitamin D intake data was estimated. The vitamin D content of foodstuffs was determined from the $6^{\text {th }}$ edition of McCance and Widdowson's The Composition of Foods ${ }^{(5)}$ and from food package labelling. Data were compared with those similarly obtained from the 4-season daily dietary records of an adult sample (20-60 years, $n=109$ completed) in Greater Manchester ${ }^{(2)}$. Adolescents $(n=110$ completed) showed little variation in vitamin D intake across the seasons. Their overall median (range) intake was only $1.92(0.01-22.15) \mu \mathrm{g} /$ day compared with $3.27(0.02-27.38) \mu \mathrm{g} /$ day in adults $(P<0.01)$. As in the adult sample, oily fish was the main food contributor and vitamin D supplements were taken by a minority $(25 \%)$ of adolescents.

We conclude that dietary vitamin D intake is very low amongst UK white adolescents and does not meet WHO recommendations ${ }^{(3)}$. It is much lower than specified in the recent IOM guidance for US and Canadian citizens ${ }^{(4)}$. Sun exposure levels and impact on vitamin D status are under study in this population, while the current data supports that within the adolescent population there is a risk that vitamin D requirements may not be met. This may have consequences for bone mineral accrual and subsequent bone health in later life.

1. Bailey DA, Faulkner RA \& McKay HA (1996) Exerc Sport Sci Rev 24, 233-266.

2. Webb AR, Kift R, Durkin MT et al. (2010) Br J Dermatol 163, 1050-1055.

3. World Health Organization (2004) Vitamin and mineral requirements in human nutrition. 2nd ed., Geneva: WHO.

4. Institute of Medicine (2011) Dietary reference intakes for calcium and vitamin D. Washington, DC: The National Academies Press.

5. Food Standards Agency (2002) McCance and Widdowson's The Composition of Foods, 6th ed., Cambridge: Royal Society of Chemistry. 